\title{
THE FLOW INSTABILITIES WITHIN AN URBAN INTERSECTION
}

\author{
Radka Kellnerová ${ }^{1,2}$ and Zbyněk Jaňour ${ }^{1}$ \\ ${ }^{1}$ Institute of Thermomechanics AS CR, Prague, The Czech Republic \\ ${ }^{2}$ Department of Meteorology and Environment Protection, Charles University, Prague, The Czech Republic
}

\begin{abstract}
The experiment uses wind-tunnel modelling method to investigate an intermittent ventilation of pollutants within an urban intersection. The model is designed after a typical inner-city area occurring frequently in European countries. It consists of apartment houses with roofs arranged to the several blocks. Blocks form regular patterns divided by intersections with height to width ratio equal to one.

Project has focused on velocity characteristics inside the intersection and adjacent canyon. The skewness and kurtosis of velocity components were studied in order to identify an area with strong intermittent tendency. Velocity measurement was carried out using 2-component LDA. Pollutants have released from line source and mean concentration was obtained using slow flame ionization detector. Comparison of time-mean flow and concentration spatial distribution verified that vertical vortices, the typical flow patterns formed inside street canyons, as well as corner vortices created just behind the turning of the street are responsible for accumulation of emissions emitted by vehicle traffic. Flow instabilities corresponding to enhancement of skewness value break down these patterns and significantly ventilate street canyons removing exhalations.

Longitudinal velocity indicates an unsteady acceleration near the rooftop level whereas deceleration occurred close to surface, so it produces a higher rotational speed in recirculation zone. In contrary, the strongest downward penetration of fresh air (into the canyon) is located behind the roof eaves on leeward building. With incoming downward gust, the recirculation zone is completely destroyed. Speed extremities (derived from kurtosis) also show the highest value at eaves level. Similar process appears in horizontal plane. Intensification of longitudinal wind runs up the circulation inside corner vortex, whereas lateral gust propagating just behind the turning of street diminishes the whole vortex and cleans up the area.

Vertical and horizontal momentum flux was analyzed by quadrant analysis. This method has detected the localities with highest momentum transfer from free stream down to the canyon. This transfer corresponds with large air mixing. It also found out notable momentum transfer from rotational zone downward to the surface, where accumulation of pollutants occurred.
\end{abstract}

Key words: Street canyon, urban intersection, skewness, quadrant analysis.

\section{INTRODUCTION}

The one of problems still keeping attention in wind-tunnel modelling is mass exchange between the polluted street canyon air and the fresh air above. The instantaneous penetrations of above-roof air into the canyon are probably responsible for intensive ventilation of exhalation from street canyon. Other way how can pollution escape from cave is to be transported under the rooftop level then inside shear stress area randomly ascend to a higher altitude and be blown away by prevailing wind. The sudden wash out in the cave is much more effective and faster method and in addition, coherent structures inside the shear layer producing gusts are currently carefully studying. During the last decade the progress was done in understanding how the aspect ratio, roof shape and presence of architectonical details affect an intensity of ventilation in canyon area. In this report we have focused on flow processes inside the urban intersection from spatial point of view.

\section{EXPERIMENTAL SET-UP}

The experiment was performed in the low-speed open-circuit aerodynamic tunnel IT AS CR in Novy Knin. Tunnel has $20.5 \mathrm{~m}$ long generating section beginning with spires and covered by roughness elements. The tunnel continues into the $2 \mathrm{~m}$ long test section with dimension of square cross-section $1.5 \mathrm{~m} \mathrm{x} 1.5 \mathrm{~m}$. Approaching flow at the entrance to the test section attains the parameters corresponding to the "very rough" boundary layer when we adopt a Snyder's classification from Guideline for fluid modelling (1981). The surface boundary is then analogous to the real downtown atmospheric conditions.

The model of urban intersection was inspired by a typical configuration of apartment houses from 19-th century, which can be frequently seen in European city-centres. The houses have 4 or 5 stages, pitched roof and they form rectangular blocks, each of them encloses a court inside. The intersections formed by blocks are mostly right-angled and in addition, they have an aspect ratio $\mathrm{H} / \mathrm{W}$ approximately equal to one.
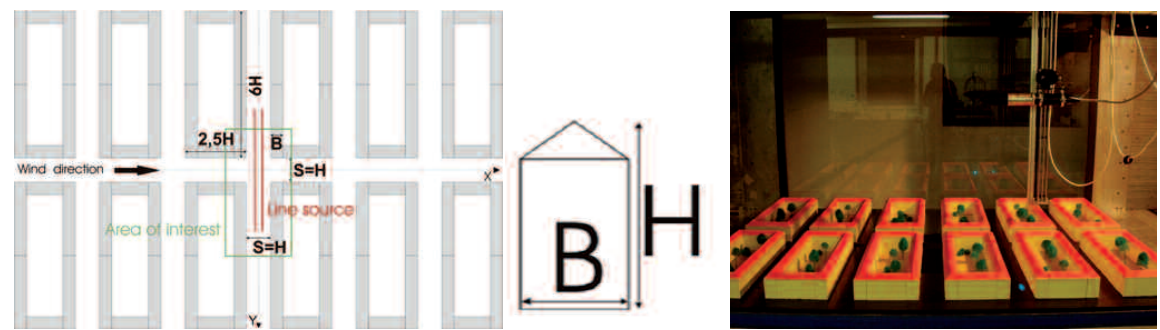

Figure 1. The scheme of model, building and photograph from the experiment. 
The scale of the model was derived from roughness length according to Jensen formula. The model is scaled down to the height of $10 \mathrm{~cm}$ with the scale factor $1: 200$. The frontal area of the model occupied 5\% of the tunnel's crosssection.

Aspect ratio $\mathrm{H} / \mathrm{W}$ between height and width of the street equals to one, while the both dimensions of building are established as $\mathrm{B} / \mathrm{H}=0.5$ for a building width and as $\mathrm{L} / \mathrm{H}=6$ for a block length (Figure 1). Hitoshi and Kimiyo used visualization to investigate how the geometry of canyon determines creation of a stable vortex. Existence of quasisteady vertical or a horizontal (so-called "corner") vortex inside the street canyon depends on the ratio between block length and street width. Considering the upcoming flow perpendicular to the street axis and unit street aspect ratio, with block length six-times larger than street width, a less more stable vertical vortex should be observed in the middle part of canyon.

The outer edges of canyon are occupied by corner vortices. Both vortex types interact together inside the interface zone what results into a final flow pattern. Kim and Baik studied a shape of recirculation zones within a cube array with RNG k-epsilon turbulence model. The aspect ratio equalled to one. On the basis of numerical study with taking into account the flow measurements from our rectangular geometry set-up (Kellnerova, 2008) we estimated a picture of final circulation in the street canyon (depicted in the Figure 2).

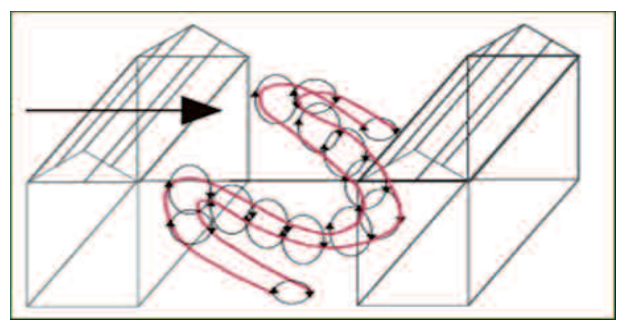

Figure 2. Estimated position of a circulation core in a canyon - scheme of the whole canyon.

Several studies concerned with street canyons have confirmed that core of vertical vortex is shifted upward with increasing ratio $\mathrm{H} / \mathrm{W}$. Theodoris and Moussiopoulos (2000) simulated numerically the flow over the canyon with a pitched roof. They suggested that dynamic of flow inside the canyon with high pitched roof were essentially different than the one we can find with a flat roof. The characteristic vertical circulation was shifted above the eaves level whereas the second counter- rotating eddy was localized near the bottom of street. Barlow (2007) recently published a paper where she proved in a wind-tunnel experiment with pitched roof houses $(\mathrm{H} / \mathrm{W}=1)$ that primary and secondary counter-vortex coexists at least during a short time interval. Long term measurement shows great reduction of velocity at street level and consequent decreasing of transfer coefficient. It started the acknowledgement that the influence of roof dimension has to be taken into account as well.

Field data obtained by Elliason et al. (2005) in Swedish Göteborg on the other side did not confirm the existence of coupled vortices for long term measurement ( 5 minutes in a full scale) but researchers were able to find out the secondary pattern when analyzing the data in short time interval (up to $5 \mathrm{~s}$ ). Nevertheless, the stacked vortices had sometimes the same rotating direction. As Elliason have concluded the lower whirl does not have to be driven by primary vortex.

Flow measurement was carried out using 2-D component Laser Doppler anemometry. Tracing particles were produced by haze generator with glycerine filling. For monitoring of reference velocity ( $\operatorname{set}$ on $4 \mathrm{~ms}^{-1)}$ we used a steel-clad transducer mounted on the tunnel axis.

The measurement error using LDA depends on the amount of detected particles. Partial average have shown that a deviation from the average value falls bellow of $1 \%$ if we detect at least ten thousand seeds. Available data rate was $100-300 \mathrm{~Hz}$ with acquisition time $100 \mathrm{~s}$. However, it was difficult to reach an optimal value at the lowest level of the canyon. For height $\mathrm{Z} / \mathrm{H}<3$ the data rate dropped to $70-100 \mathrm{~Hz}$ due to low velocity and the lack of glycerine fog beyond the obstacles. The total error was estimated from repeated velocity measurements at the same point and with the same tunnel conditions. The error remains $3.5 \%$ for the $U$-velocity, but reaches $8 \%$ for the $W$-velocity because of vertical component smaller average value.

\section{RESULTS \\ Vertical plane}

The general description of recirculation zone in the canyon consists from primary vertical vortex, which dominates in the pollution transportation from the ground level into the stream flow. This recirculation produces the typical enhanced sleeve of high mean concentration on the leeward side, while the windward side is successfully ventilated 
and concentration attains only 1/10th of leeward value. Local maximum often takes place near the foot of the leeward side, whilst the minimum is centralized in the middle of canyon.

When the secondary vortex is strong enough, local maximum can exist also near the base of windward side. The secondary whirls are hardly detectable with LDA method because of their position too close to ground.

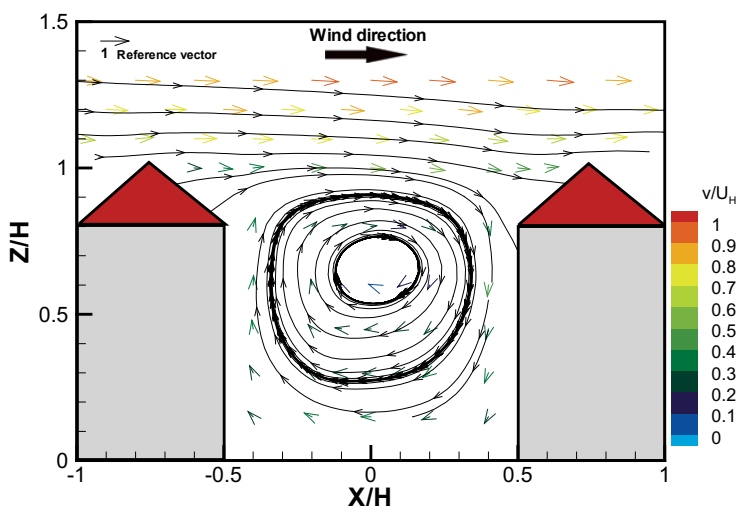

Figure 3. The reference picture of a flow pattern inside a canyon. $\mathrm{Y} / \mathrm{H}=1.5 . \mathrm{U}_{\mathrm{H}}$ is velocity at $\mathrm{Z}=\mathrm{H}$.

As could be seen in Figure 2, the vortices are changing with the decreasing distance from the centre of canyon to the edge. The core of the vertical vortex reaches its highest altitude at the lateral distance $\mathrm{Y} / \mathrm{H}=1.5$. The situation in vertical cross-section in this distance is plotted in the Figure 3. The data are gained from 100s long time records.

In a turbulent time scale, the main vortex changes its shape or position, the rotation is accelerated or decelerated as the air from the roof level propagates into the cave. Information about intermittent motion can offer plots with spatial distribution of skewness (Fig. 4). From the picture for longitudinal velocity can be clearly seen how upper half of vortex is temporarily accelerated. The maximum of positive values is located just behind the rooftop. The acceleration in opposite direction occurs in the lower half of vortex, so together they produce a higher rotational speed in recirculation zone. The second picture confirms the well-known idea about occasional penetration of clear air into the canyon. The area where intermittent downward movements play a role is spread over the whole crosssection at the height of roof. The extreme value is located behind the leeward eaves. This induced an expectation that vortex can be broken or disturb by incoming gust and consequently the street can be for a few seconds cleaner. Although that plot suggests the above-roof air can break the vortex completely, on visualization this happens very rarely. The clear air more likely enters the cave from downwind half of street. The circulation is after pressed and stretched against the upwind side and upwards but not stopped.

a)

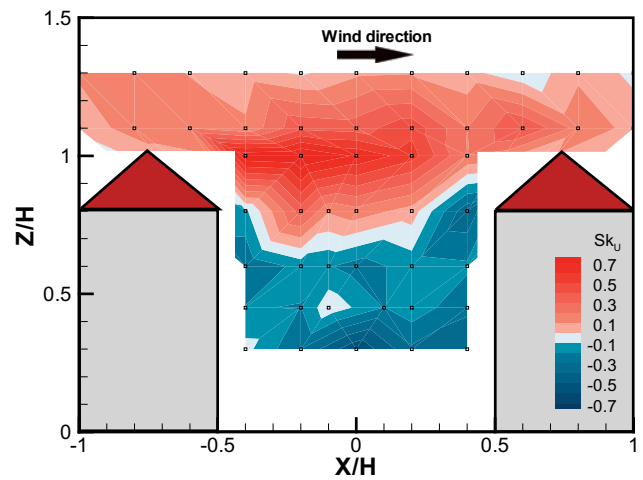

b)

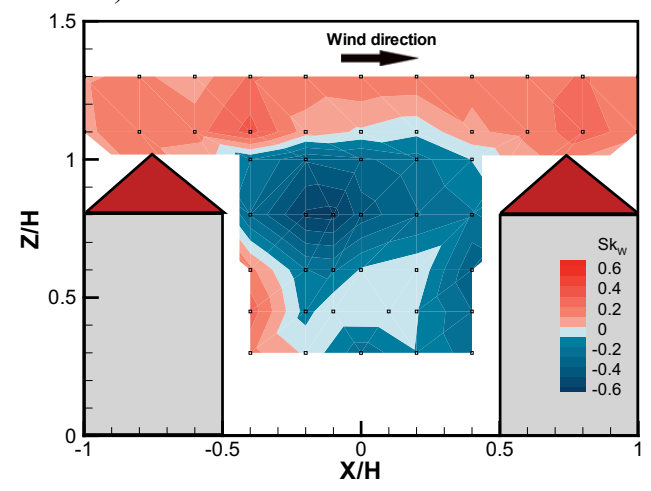

Figure 4. a) Skewnes of $U$-velocity. b) Skewness of $W$-velocity. $\mathrm{Y} / \mathrm{H}=1.5$.

Other high values of negative skewness can be found on the windward wall where they participate on the acceleration of vortex rotation via vertical component. This matches to positive skewness at the leeward wall. For both components speed extremities derived from kurtosis also show the highest value at eaves level.

Barlow (2007) compared the profiles of skewness for flat roof canyon and for high pitched roof set-up (roof takes $33 \%$ of the building height). She explained the displacement of vortex core upwards and poor ventilation in a saddle 
roof case as an influence of roof shape on flow dynamics. For street aspect ratio $\mathrm{H} / \mathrm{W}=1$, it could be seen either on visualization either in her plots of skewness where asymmetrical maximum completely vanished.

Our case has a pitched roof taking only $20 \%$ of building height and unit street aspect ratio, and no evidence about such different dynamics was found. Visualization did not show any stable counter-rotating vortex, LDA measurement proved the presence only single main circulation and skewness analysis confirmed the enhanced intermittent downdraughts on the leeward side. If we establish so-called "roof aspect ratio" when dimension of roof R is divided by width of street, then seems to be significant difference in dynamics around the canyon for roof aspect ratio $\mathrm{R} / \mathrm{W}=0.2$ and for 0.33 .

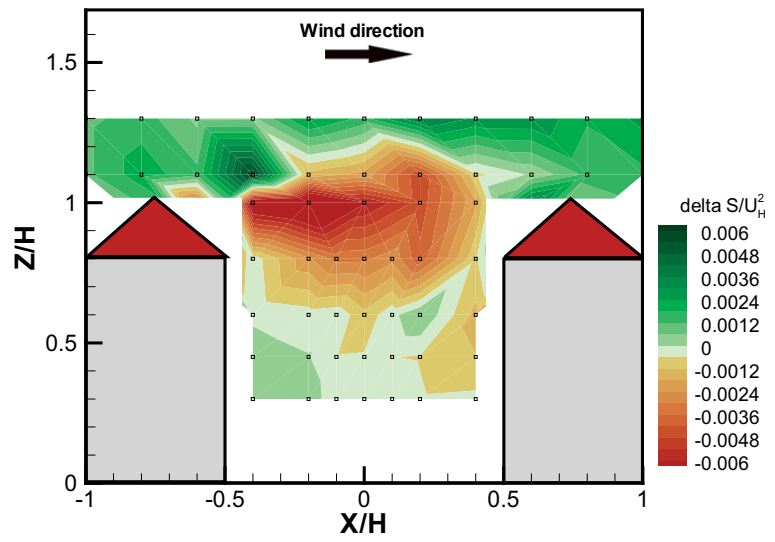

Figure 5. The difference in flux momentum between sweep and ejection (both negative). $\mathrm{Y} / \mathrm{H}=1.5$. The red colour corresponds with domination of sweep. Green colour marks domination of ejection.

While vertical velocity distribution shows the faster downdraughts and longitudinal velocity indicates short-time acceleration at the rooftop level, it could be a question whether these two-component fluctuations are correlated. Since the negative mean momentum flux is typically located near a roof, superposition of two components in this location can interpret the local extreme of sweeps or ejections.

Quadrant analysis was applied on flow time series to receive particular contributions of momentum flux from particular quadrants. The sweep and ejection occur exclusively above the eaves level with noticeable extreme just behind the rooftop of leeward building. Positive portion of momentum flux are centralized in the lower half of canyon except one point just above the rooftop. In order to recognize whether the skewness of velocity distribution is connected rather with sweeps than with ejections, we subtracted the ejections from the sweeps. The result plot is depicted in Figure 5.

The momentum of sweep dominates to ejection over whole roof area. The maximum is located at point where the longitudinal skewness reaches its extreme as well. The high $U$-velocity fluctuations help to increase sweep flux. Furthermore, moderately strong energy transport covers larger area then longitudinal skewness can explain. If follows, in lower roof area, Reynolds stress must be supplied by an energy from vertical intermittent descending movements. Slightly above the rooftop level, ejections start to be major contributor to the momentum flux.

\section{Horizontal plane}

Similar process appears in horizontal plane. The reference flow situation inside an intersection is shown in Figure $6 \mathrm{a}$. The velocity field was measured at $\mathrm{Z} / \mathrm{H}=0.3$. Intensification of longitudinal wind runs up the circulation inside corner vortex (Fig. 6b), whereas lateral gust propagating just behind the turning of street tries to diminish the whole vortex and cleans up the area (Fig. 6c).

On the contrary from vertical cross-section, lateral propagation behind the corner is much weaker then disturbances near the roof eaves. The more stable corner vortex structure was confirmed by visualisation as well. It would be noted that other dissimilarity in comparison with vertical section is absence of any accelerating force by lateral velocity inside intersection. Instead of this, the lateral component indicates the effort to decelerate the wind divergence on the windward corners.

Since the recirculation zone evolves with the fetch from central intersection we investigated how also intermittent flow structure can depend on the distance from central intersection. The vertical cross-section at the distance $\mathrm{Y} / \mathrm{H}=0.5$ (corner). 0.75 (centre of corner vortex), 1 and 1.5 (highest position of vortex core) was measured. Surprisingly no noticeable change in the vertical skewness and kurtosis was found. Although the concentration inside the corner vortices is more than three time higher and the profile of concentration is definitely distinct from profile in crosssection at $\mathrm{Y} / \mathrm{H}=1.5$ (Kellnerova, 2007), intermittent penetrating is identical along whole canyon. It means that at distance $\mathrm{Y} / \mathrm{H}=0.75$ is not weaker penetration what can help to form corner vortices. The formation of these vortices is 
fully driven by street flow (up to height of eaves in the case of pitched roof), depends strongly on geometry and not on the locally unique dynamics above the roof.

a)

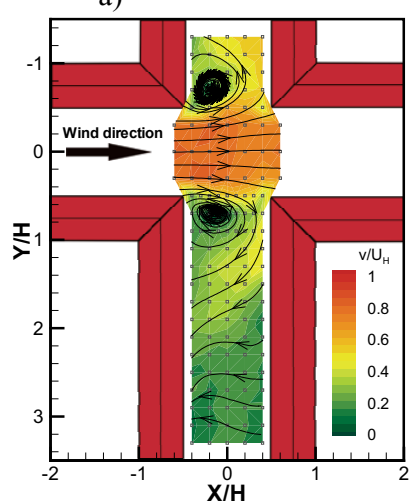

b)

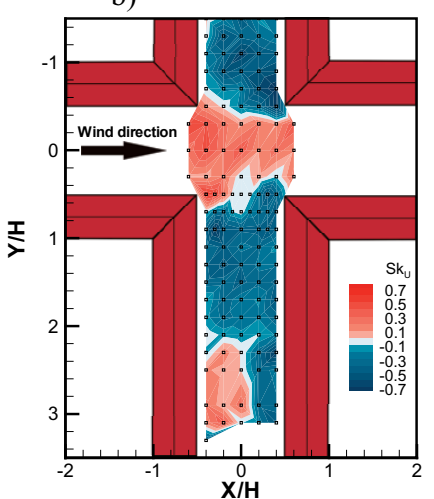

c)

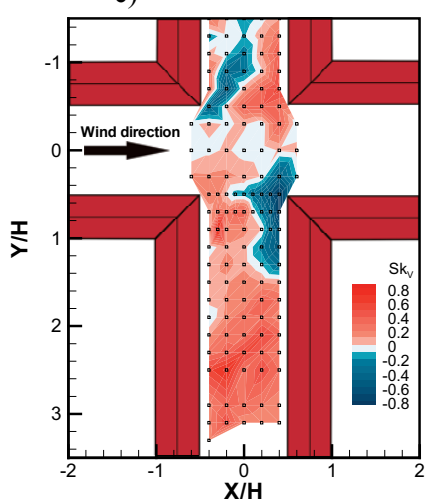

Figure 6. a) The reference velocity field, $\mathrm{U}_{\mathrm{H}}$ is velocity at $\mathrm{Z}=\mathrm{H}$. b) Skewness of $U$-velocity. c) Skewness of $V$-velocity.

\section{CONCLUSION}

The experiment about flow instabilities inside a street canyon was conducted in slow-speed wind tunnel. Flow measurement was performed on the model of urban intersection inspired by European inner city. The buildings have a pitched roof with roof aspect ratio $\mathrm{R} / \mathrm{W}=0.2$. For this ratio no particular change in flow pattern from long-time point of view was found. Mean skewness in distribution of longitudinal velocity clarifies the tendency for occasional running up the circulation. The mean vertical skewness confirms the presence of intermittent downdraught near the leeward eaves and also near the windward wall. The first is responsible for disturbing main recirculation zone. The latter helps to accelerate rotational speed of vertical vortex. Visualization shows that fully destruction of vortex happens very rarely. Vortex is rather bended and stretched how the clear air presses downwards.

Comparison between spatial distribution of skewness and "ejection subtract from sweep" momentum flux shows that sweep are supplied better with large positive U-fluctuations at to rooftop level, whereas better with negative Wfluctuations at the eaves level.

In horizontal plane the lateral gust propagation is weaker than in the vertical case. The lateral component does not participate on the spinning up of corner vortices. Thus the corner vortices are much more stable then the vertical vortex. No noticeable changes in vertical skewness and kurtosis were found out along the canyon axis. The accumulation of pollution inside the corner vortices is fully controlled by low street wind and no by special local flow processes at the rooftop level.

Acknowledgements: Authors thank for the support from A VOZ20760514.

\section{REFERENCES}

Barlow, J.F. and B. Leitl, 2007: Effect of Roofshape on Unsteady Flow Dynamics in the Street Canyons. Internat. Workshop on Physical Modelling of Flow and Dispersion Phenomena, Orléans, France, 75-78.

Eliassona, I., B. Offerlea, C.S.B. Grimmondb and S. Lindqvista, 2006: Wind Fields and Turbulence Statistics in an Urban Street Canyon. Atmospheric Environment, 40, 1-16.

Kellnerova, R., M. F. Yassin and Z. Janour, 2007: Wind-Tunnel Modelling and Numerical Simulation Within Urban Intersection. Internat. Workshop on Phy. Modell. of Flow and Disper. Phenomena, Orléans, 231-235.

Kellnerova, R. and Z. Janour, 2008: Symmetry of Laser Doppler Anemometer Measurement in Wind-Tunnel Modeling. Acta technica CSA $V, \mathbf{5 3}$, (in print).

Kim, J. J. and J. J. Baik, 2004: A Numerical Study of the Effects of Ambient Wind Direction on Flow and Dispersion in Urban Street Canyons Using the RNG k-epsilon Turbulence Model. Atmosph. Env., 38, 3039-3048.

Kono, H. and K. Kusunoki, 2004: 3-D Stream and Vortexes in an Urban Canopy layer and Transport of motor vehicle exhaust gas - Wind Tunnel Experiment. 9th Int. Conf. on Harmonisation within Atmospheric Dispersion Modelling for Regulatory Purposes, 70-74.

Theodoridis, G. and N. Moussiopoulos, 2000: Influence of building density and roof shape on the wind and dispersion characteristics in an urban area: A numerical study. Environ. Monit. Assess., 65, 407-415.

Snyder,W.H., 1981: Guideline For Fluid Modeling Of Atmospheric Diffusion. Meteorology ond Assessment Division Enviromental Sciences, U.S. Enviromental Protection Agency. 